\title{
TURBULENCE IN THE ONE-DIMENSIONAL COMPLEX GINZBURG-LANDAU EQUATION
}

\author{
A. ZUBRZYCKI \\ Department of Physics, Technical University \\ Malczewskiego 29, 26-600 Radom, Poland
}

(Received November 8, 1994; revised version January 30, 1995)

The transition to phase and amplitude turbulent states in the one-dimensional complex Ginzburg-Landau equation is investigated by a numerical simulation. In order to describe existing attractors quantitatively, the largest Lyapunov exponent is estimated. The largest Lyapunov exponent is positive but small for the phase turbulence and it is much larger for the amplitude turbulence. Moreover, it seems independent on the system size.

PACS numbers: 05.45.+b

One of the simplest models of spatially extended dissipative systems showing spatio-temporal chaos is the one-dimensional complex Ginzburg-Landau equation [1]

$$
A_{t}=\mu A-(1+\mathrm{i} \alpha)|A|^{2} A+(1+\mathrm{i} \beta) A_{x x} .
$$

It describes a small and slowly varying complex field $A(x, t)$, called an order parameter, which is a measure of an oscillatory instability of a one-dimensional medium in the vicinity of a Hopf bifurcation [2]. Equation (1) has been investigated recently by some authors and there has been considerable progress in understanding its chaotic dynamics [3-5]. It has been found that there are at least two types of a chaotic state in this system. The first is the "phase turbulent" state with the field magnitude $|A|$ almost constant in space and time. The threshold of the phase turbulence in the $(\alpha, \beta)$ parameters space is the zero value of $1+\alpha \beta$. The phase turbulence appears for negative $1+\alpha \beta$. On the other hand as long as $1+\alpha \beta$ is positive a homogeneous solution of $\mathrm{Eq}$. (1) for $\mu>0$,

$$
A(t)=\sqrt{\mu} \exp (-\mathrm{i} \alpha \mu l),
$$

is linearly stable. The second chaotic state is the "amplitude turbulent" state, called also a "defect chaos", involving large amplitude fluctuations and isolated "defects" with the zero field $A$ value. The aim of this paper is to investigate by the numerical simulation a process of the transition to the phase and amplitude turbulence. In order to describe quantitatively the chaotic state in the two phases, the largest Lyapunov exponent was determined. A still open question is whether 
these two states are really distinct in the thermodynamic limit of the infinite system. So as to try to answer this question the largest Lyapunov exponent was examined in dependence on the system size.

Equation (1) was solved numerically in the domain $-L \leq x \leq L$ with periodic boundary conditions. A pseudo-spectral method [6] was used for space, and a 3-th order Adams-Bashforth-Moulton predictor-corrector method with a fixed time step for time discretization. As an initial condition a random initial condition was chosen. In order to find whether some kind of extrapolation of the results to the thermodynamic limit $L \rightarrow \infty$ is possible, the calculations were performed for different system sizes $2 L$ (of the order of $10^{3}$ ) and then for different numbers of mesh points in space $n$ (up to 512). It was found that the results depend on the ratio $\Delta x=2 L / n$ rather than on the system size itself. In order to compare the results with the recent trial of the estimation of the largest Lyapunov exponent for the two-dimensional Ginzburg-Landau equation by Bohr et al. [7], the same configuration of the $\mu, \alpha, \beta$ parameters were used. The values of $\mu(=0.2)$ and $\beta(=-1)$ parameters were kept fixed while $\alpha(>0)$ was varied. One way to find the largest Lyapunov exponent was to measure directly a rate of the time divergence of two initially close trajectories of the attractor. A given post-transient configuration $A(x, t)$ was slightly perturbed by adding a small quantity $\delta A$ (typically $10^{-15}$ ) to the real part of $A(x=0, t)$. Then the evolution of both the perturbed $\left(A^{\prime}\right)$ and unperturbed $(A)$ systems was followed and their "distance" at equal times was measured. As this distance

$$
\Delta S(t)=\sqrt{\sum_{i=1}^{n}\left|A^{\prime}\left(x_{i}, t\right)-A\left(x_{i}, t\right)\right|^{2}}
$$

was taken [7], where $n$ is a number of mesh points and $x_{i}=(2 i / n-1) / L$. The largest Lyapunov exponent emerges as the slope of $\ln \Delta S$ versus time. The Lyapunov exponent obtained by this method is always a "finite-time" Lyapunov exponent because one can get a positive slope of $\ln \Delta S$ only up to some transient time $T$. In order to verify that the value of this "finite-time" Lyapunov exponent is equal to the value of "real" Lyapunov exponent, the latter was obtained using the standard method of Benettin et al. [8]. In this method the Lyapunov exponent is derived from the solution of the tangent equation, that is, the equation linearized about the investigated $A(x, t)$ :

$$
\psi_{t}=\mu \psi-(1+\mathrm{i} \alpha)\left(2|A|^{2} \psi+A^{2} \psi^{*}\right)+(1+\mathrm{i} \beta) \psi_{x x}
$$

where $\psi$ is an $n$-dimensional tangent vector and $\psi^{*}$ is its complex conjugate. The comparison of the results obtained by these two methods and other arguments for equivalence of the "finite-time" and "real". Lyapunov exponents will be given later.

Firstly, the general features of the complex Ginzburg-Landau system will be shown. As long as $\alpha \leq 1.0$, i.e. below the threshold of the phase turbulence, the system evolves approaching the homogeneous solution (2). It is seen in Fig. 1a, which shows a pattern of the system after a long time evolution $(t=15000$ while the transient time needed to reach the attractor is about 1500) for $\alpha=1.0$. The amplitude of $A$ is constant in the whole space and close to $\sqrt{\mu} \approx 0.447$. The 
(a)

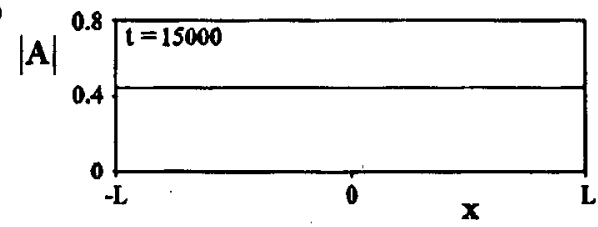

(b)

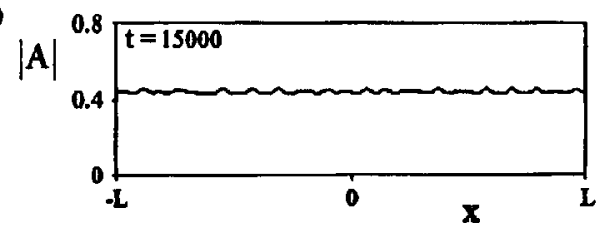

Fig. 1. The patterns of the amplitude of $A$ after a long time evolution of the system for (a) $\alpha=1.0$, (b) $\alpha=1.39$. The elapsed time is indicated in the top left hand corners. $L \approx 300$.

(a)

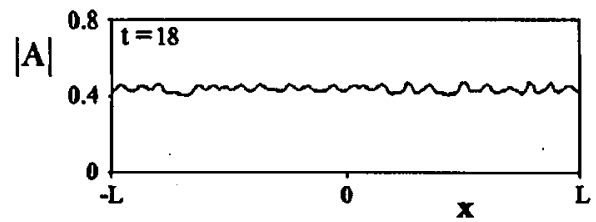

(b)

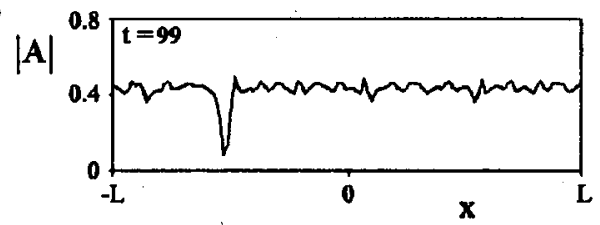

(c)

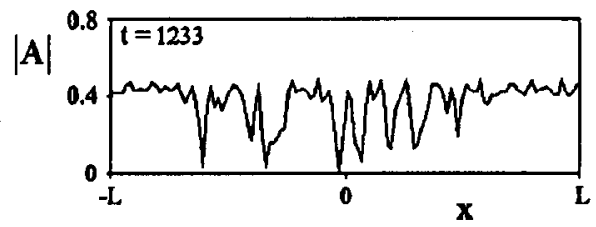

(d)

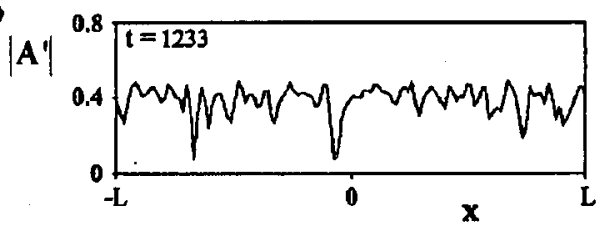

Fig. 2. (a,b,c) Three snap-shot patterns of the amplitude of $A$ at various moments of time indicated in the top left hand corners. (d) A picture corresponding to Fig. $2 \mathrm{c}$ obtained for a slightly different initial condition $\left(\operatorname{Re} A^{\prime}(x=0, t=0)=\operatorname{Re} A(x=0, t=0)+\right.$ $10^{-15}$ ). In all cases $\alpha=2.0$ (amplitude turbulence regime) and $L=300$. 

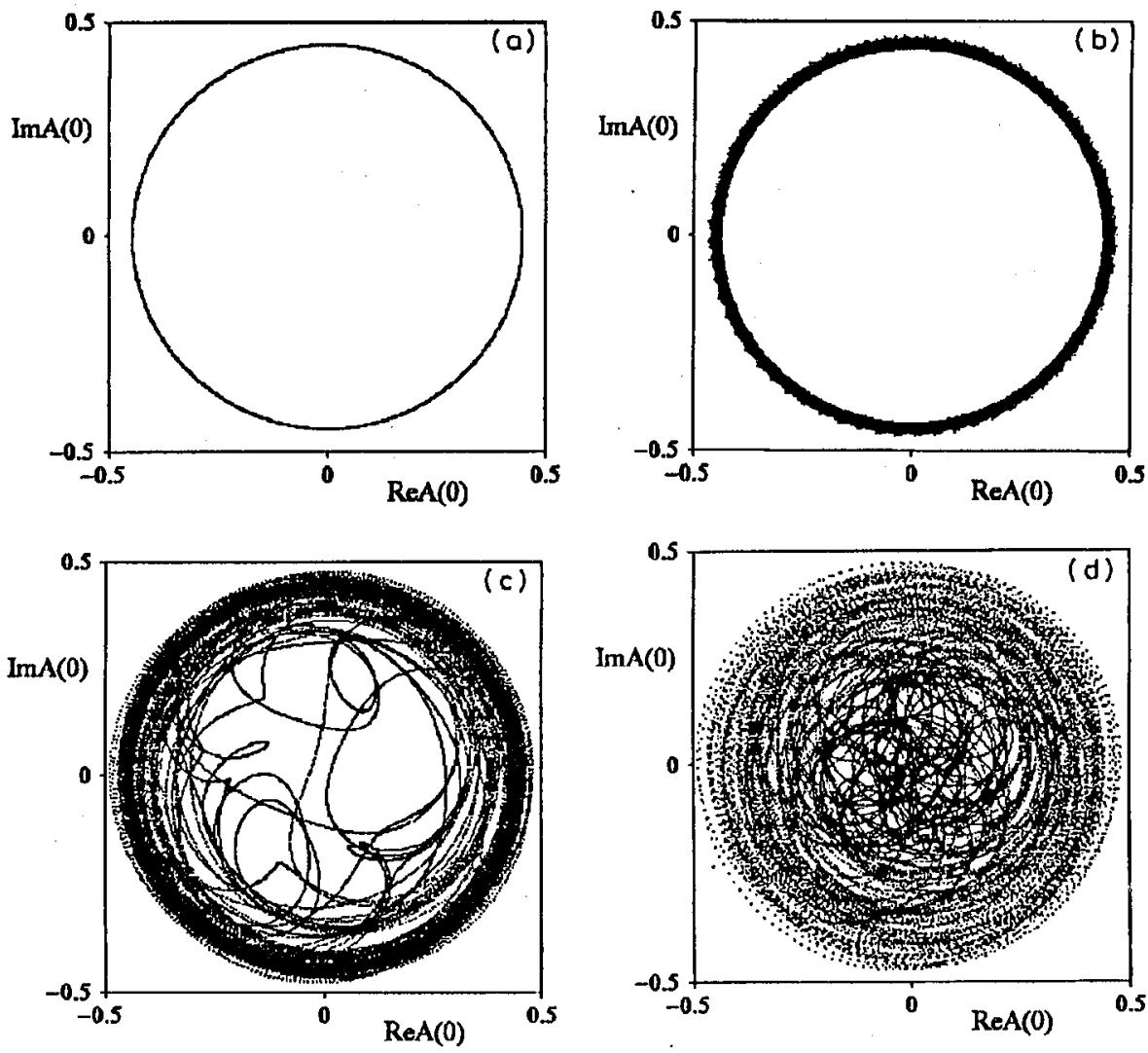

Fig. 3. Post-transient phase portraits $\operatorname{Im} A(0)$ versus $\operatorname{Re} A(0)$ for (a) $\alpha=1.0$, (b) $\alpha=1.39$, (c) $\alpha=1.40$, (d) $\alpha=3.0$. Each portrait contains 32000 points and the time between successive data is 0.09 .

corresponding pliase portrait $\operatorname{Im} A(0)$ versus $\operatorname{Re} A(0)$ is a circle of the radius close to $\sqrt{\mu}$ (Fig. 3a), and the phase points run round the circle with a frequency $\omega \approx \alpha \mu$. In the region of the phase turbulence for $1.0<\alpha<1.4$ the final pattern of the amplitude exhibits small fluctuations around $\sqrt{\mu}$ and the corresponding phase portrait changes from a circle into a narrow strip (Figs. $1 \mathrm{~b}$ and $3 \mathrm{~b}$ for $\alpha=1.39$ ). For $\alpha \geq 1.4$ the system approaches the amplitude turbulent state. Figures $2 \mathrm{a}-2 \mathrm{c}$ show the evolution of the system for $\alpha=3.0$. At the beginning, the system passes through a state with small fluctuations of the amplitude like in the phase turbulence, but after some time a shock-like structure appears. The appearance of such structures within a background of small amplitude fluctuations always indicates the onset of the amplitude turbulent state. Figure $2 \mathrm{c}$ shows the post transient state with the large amplitude fluctuations. Figure $2 \mathrm{~d}$ was obtained for the initial condition that differed very slightly (by $10^{-15}$ ) at one point of the space from this for Figs. 2a-2c. From the comparison of Fig. 2c and Fig. 2d strong sensitivity to initial conditions is seen. It is a common feature of a chaotic 
behaviour. Figures $3 \mathrm{c}-3 \mathrm{~d}$ show the phase portraits $\operatorname{Im} A(0)$ versus $\operatorname{Re} A(0)$ of the chaotic attractors for the threshold value $\alpha=1.40$ and well above this value $\alpha=3.0$.

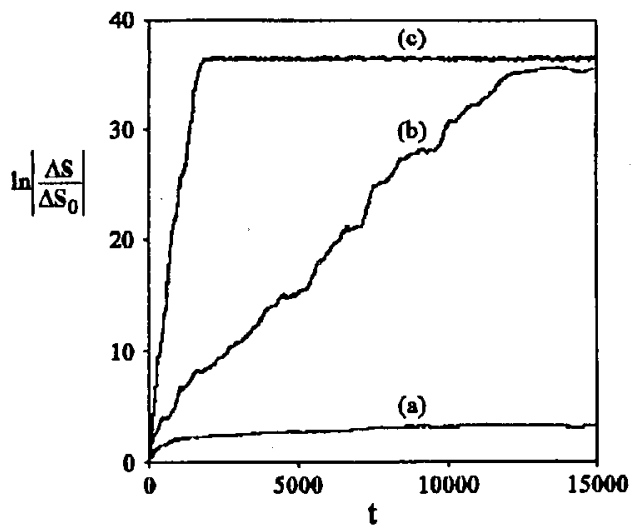

Fig. 4. Time dependence of $\ln \left|\Delta S / \Delta S_{0}\right|$, (where $\Delta S_{0}=\Delta S(t=0)$ ) for three different values of $\alpha$ : (a) $\alpha=1.0$, (b) $\alpha=1.39$, (c) $\alpha=1.40$. The point of the transition to the amplitude turbulence lies between $\alpha=1.39$ and $\alpha=1.40$.

As it was mentioned the transition to the amplitude turbulent state takes place for $\alpha \approx 1.40$. It is seen from the comparison of the phase portraits for $\alpha=1.39$ and $\alpha=1.40$. This transition is also reflected by the behaviour of the "finite-time" Lyapunov exponent. Figure 4 shows the dependence of $\ln \Delta S$ on time, where $\Delta S$ is defined by Eq. (3), for three different values of $\alpha=1.0,1.39$ and 1.40. The "finite-time" Lyapunov exponent, which emerges as the slope of $\ln \Delta S$, is practically equal to zero for $\alpha=1.0$, very small $(\approx 0.003$ ). for $\alpha=1.39$ but it is much larger $(\approx 0.02)$ for $\alpha=1.40$.

The summary of the features of the "finite-time" Lyapunov exponent is given in Fig. 5. Figure 5a shows the dependence of $\ln \Delta S$ on time for three different values of $\alpha=1.75,2.0,2.5$ (being in the amplitude turbulence regime) while the initial difference between trajectories under investigation $\delta A$ is equal to $10^{-15}$. Figure $5 \mathrm{~b}$ shows the same dependence for three different values of $\delta A=10^{-5}$, $10^{-10}, 10^{-15}$ and $\alpha=3.0$. Note that there is always the transient time after which the slope of $\ln \Delta S$ becomes equal to zero and a "saturation" value of $\ln \Delta S$ is independent on $\alpha$ but dependent on $\delta$. For a given $\alpha$ the transient time becomes longer with decreasing $\delta A$, while the slope of $\ln \Delta S$ is almost the same for every (small enough) $\delta A$, so assuming $\delta A \rightarrow 0$, the slope of $\ln \Delta S$ can be extrapolated to infinite time and it gives the "true" Lyapunov exponent. This conjecture was confirmed by the computation of the Lyapunov exponent from the solution of the tangent equation (4). Figure 6 gives the comparison of the results. It shows the largest Lyapunov exponent as a function of the $\alpha$ parameter. The Lyapunov exponent estimated as the slope of $\ln \Delta S$ is marked with circles while the Lyapunov exponent obtaincd from the solution of the tangent equation with crosses. The 

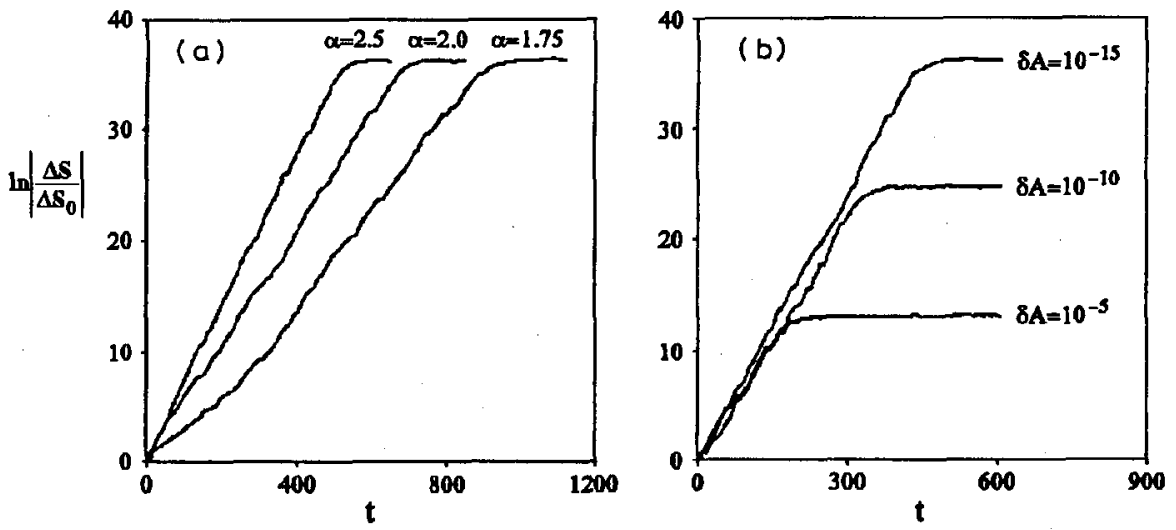

Fig. 5. Time dependence of $\ln \left|\Delta S / \Delta S_{0}\right|$, (where $\Delta S_{0}=\Delta S(t=0)$ ) for (a) three different values of $\alpha=1.75,2.0,2.5\left(\delta A=10^{-15}\right)$, (b) three different values of $\delta A=$ $10^{-5}, 10^{-10}, 10^{-15}(\alpha=3.0)$. All values of $\alpha$ lie in the amplitude turbulence regime.

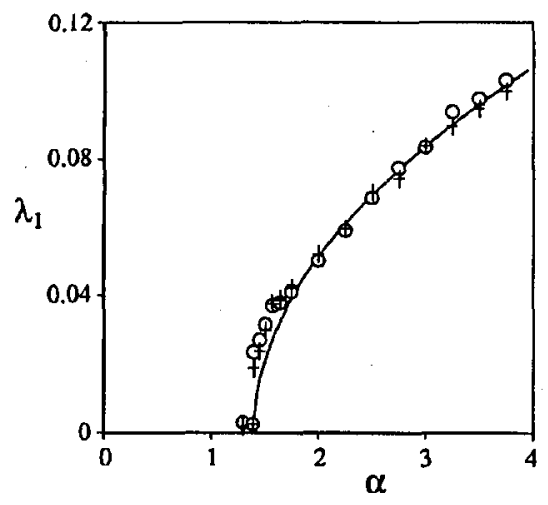

Fig. 6. The largest Lyapunov exponent $\lambda_{1}$ as a function of the $\alpha$ parameter. Circles were obtained as the slope of $\ln \left|\Delta S / \Delta S_{0}\right|$ versus time, while crosses from the solution of the tangent equation (4). The solid curve is a power-law fit (5) with $\eta=0.066, \nu=0.506$ and $\alpha_{t}=1.40$.

solid line in Fig. 6 is a power-law fit describing the largest Lyapunov exponent as a function of $\alpha$ for $\alpha>1.75$ :

$$
\lambda_{1}=\eta\left(\alpha-\alpha_{t}\right)^{\nu},
$$

where $\alpha_{t}$ is the value of $\alpha$ for which the transition to the amplitude turbulence occurs. This dependence was checked for a number of random initial conditions and the values of $\eta, \nu$ were found as being approximately equal to $\eta \approx 0.06 \div 0.07$ and $\nu \approx 0.5 \div 0.6$ (in two dimensions $\eta=1.0$ and $\nu \approx 0.5[7]$ ).

It must be emphasized that the terms "finite-time" Lyapunov exponent and "transient time" used here, relate only to the particular method of the estimation of the Lyapunov exponent as the slope of $\ln \Delta S$ versus time and they 
have different meaning than those applied by Bohr et al. They have found that in two-dimensional complex Ginzburg-Landau system there is a range of $\alpha$ for which the amplitude turbulent state is transient. In this range one can assign the "finite-time" positive Lyapunov exponent only up to some transient time after which the amplitude turbulence vanishes, while the "true" largest Lyapunov exponent assigned for infinite time is equal to zero. In the present simulation there has not been found any example of the amplitude turbulent state being transient.

The threshold of the amplitude turbulence as well as all the results shown in Figs. 1-6 were obtained for the same random initial condition $A(x, t=0)$ and the same system size $2 L \approx 600$ (with $n=128$ mesh points). A discussion of the influence of the initial conditions and the size of the system on the results is necessary here. It has been found that there is a hysteretic range [4] for $\alpha$ lower than approximately 1.75 . In this range the system evolves towards the attractors which are dependent not only on $\alpha$ but also on initial conditions. In particular, the point of the transition to the amplitude turbulence $\alpha_{t}$ lies always in this range, so it strongly depends on initial conditions. For $\alpha>1.75$ it seems that the solutions converge to the chaotic attractors which are dependent only on $\alpha$, as the value of the estimated Lyapunov exponent does not depend on initial conditions. As regards the dependence on the system size, Fig. 7 shows the largest Lyapunov exponent computed for $\alpha=3.0$, far above the hysteretic range, as a function of the distance between mesh points $\Delta x=2 L / n$. This figure was obtained for $n=128$, 256,512 mesh points. It means that for a given $\Delta x$ the estimated values of the Lyapunov exponent correspond to three consecutively doubled system sizes. The results are similar in these three cases and the value of the Lyapunov exponent is almost constant in a range of $\Delta x$ between approximately 4.0 and 6.0 (for a fixed time step $\Delta t=0.36)$. For a smaller $\Delta x$ errors appear related to weak stability of the numerical scheme and when $\Delta x$ is too large the numerical scheme becomes a poor truncation of (1), but it is believed that the results are credible in the mentioned range. The results shown in Fig. 7 suggest that the value of the estimated
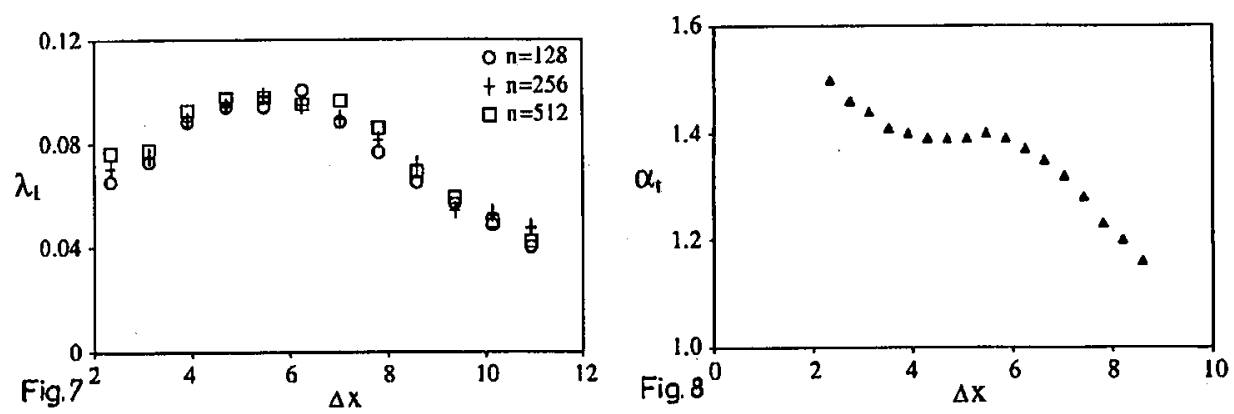

Fig. 7. The largest Lyapunov exponent $\lambda_{1}$ as a function of the distance between mesh points $\Delta x$ for three different numbers of mesh points $n=128,256,512$.

Fig. 8. The point of the transition to the amplitude turbulence $\alpha_{t}$ as a function of the distance between mesh points $\Delta x$. 
Lyapunov exponent does not depend on the system size, provided it is properly chosen for a numerical simulation. Figure 8 shows a dependence of the point of the transition to the amplitude turbulence $\alpha_{t}$ on the distance between mesh points $\Delta x$ for the same initial condition as for Figs. 1-6. There is again the range of $\Delta x$ between approximately 4.0 and 6.0 , in which the estimated value of $\alpha_{t}$ is almost constant.

\section{Conclusion}

In summary, the numerical simulation of the one-dimensional complex Ginzburg-Landau system was performed and the transition to the phase and amplitude turbulence was investigated by varying the imaginary part of the nonlinearity coefficient $\alpha$. It has been found that one can distinct three different regimes of possible chaotic attractors with increasing $\alpha$. The first is the phase turbulence regime in which the largest Lyapunov exponent is small. The second is the hysteretic (bichaotic) regime, in which it depends on initial conditions, whether the system ends up in the phase or amplitude turbulent state. It should be noted that the precise border between the phase turbulence and hysteretic regimes has not been determined. The third one is the amplitude turbulence regime appearing for large enough $\alpha$. In this regime all attractors found depend on the value of $\alpha$ only and not on the initial conditions. The largest Lyapunov exponent is about an order of magnitude larger for the amplitude than for the phase turbulent state. The important conclusion from the present simulation is independence of the Lyapunov exponent and then of the possible attractors on the system size. By extrapolation, as there is a considerable difference between the value of the largest Lyapunov exponent estimated for the amplitude and phase turbulence, this independence suggests that the two phases are really distinct in the thermodynamic limit.

\section{Acknowledgment}

I would like to thank Prof. Andrzej Sukiennicki for careful reading of the manuscript and many positive remarks.

\section{References}

[1] H. Haken, Synergetics, An Introduction: Nonequilibrium Phase Transition and Self-Organization in Physics, Chemistry, and Biology, Springer, Berlin, Heidelberg, New York 1983; Y. Kuramoto, Chemical Oscillations, Waves, and Turbulence, Springer-Verlag, Berlin, Heidelberg, New York, Tokyo 1984.

[2] A.C. Newell, Lect. Appl. Math. 15, 157 (1974).

[3] H. Sakaguchi, Prog. Theor. Phys. 83, 169 (1990); ibid 84, 792 (1990).

[4] B.I. Shraiman, A. Pumir, W. van Saarloos, P.C. Hohenberg, H. Chate, M. Holen, Physica D 57, 241 (1992).

[5] D.A. Egolf, H.S. Greenside, Nature 369, 129 (1994); Phys. Lett. A 185, 395 (1994).

[6] B. Fornberg, G.B. Whitham, Philos. Trans. R. Soc. 289, 373 (1978).

[7] T. Bohr, A.W. Pedersen, M.H. Jensen, Phys. Rev. A 42, 3626 (1990).

[8] G. Benettin, L. Galgani, A. Giorgilli, J.-M. Strelcyn, Meccanica 15, 21 (1980). 